\title{
SOME CRITERIA FOR THE DISAPPEARANCE OF THE MUSHY REGION IN THE STEFAN PROBLEM
}

\author{
BY
}

I. G. GÖTZ (Institute of Applied Mathematics and Statistics, Technical University of Munich, Germany)

AND

B. ZALTZMAN (CEEP, Blaustein Institute for Desert Research, Ben-Gurion Univ. of the Negev, Israel)

Abstract. The disappearance of the mushy region in a multidimensional one-phase Stefan problem is discussed. In the case of a piecewise-smooth boundary of the domain and bounded initial-boundary data, sufficient conditions for the disappearance of the mushy zone in a finite time are presented. For a $C^{2}$-smooth boundary and appropriately smooth boundary data both necessary and sufficient conditions for the mush to vanish are obtained. Possible behaviors of the transient phase for a twodimensional solution near a corner point of the domain are also investigated.

1. Introduction. It is well known that the enthalpy formulation of the Stefan problem, investigated first by Oleinik [12] and Kamenomostskaya [6], sometimes yields the appearance of a finite region, entirely at the melting temperature. The nonlinear heat equation degenerates in this zone, and the features of this degeneration determine the behavior of the transient phase. The appearance of this transient set was observed first in the numerical calculations by Atthey [1], and he also gave it the name of "mushy region", widely used now. Meirmanov [8] and Primicerio [13] proved that the enthalpy formulation of the Stefan problem in the presence of internal heat sources with appropriate initial data yields a mushy region.

The evaluation of the lifetime of a mush has been initiated by Meirmanov [9] for the one-dimensional Stefan problem. He proved that, provided some energy criterion holds, the mushy zone disappears in a finite time. Otherwise, it exists for all times. He obtained also a similar result for spherically-symmetric solutions of the Stefan problem (Meirmanov [10]).

The first attempt to investigate the behavior of the mushy region in a multidimensional two-phase Stefan problem was made by Rogers and Berger [14]. They proved that the mushy region does not increase in the homogeneous Stefan problem with constant Dirichlet data and almost uniformly continuous initial data. These results were generalized in [4] where it was shown that for an arbitrary bounded solution of the Stefan problem, the mushy region is nonincreasing in time in the set-theoretical

Received January 22, 1993.

1991 Mathematics Subject Classification. Primary 80A22, 35K05.

(C)1995 Brown University 
sense. This result holds for an inhomogeneous Stefan problem under some conditions on the behavior of the heat source in the mushy region.

The measure of the mushy region in the one-phase multidimensional Stefan problem has been estimated by Gustafsson and Mossino [5] with the help of a refined technique of isoperimetric inequalities, by assuming a constant temperature $\boldsymbol{\theta}$ on each of the two connected components of the boundary: $\Theta>0$ on the first, $\Theta=0$ on the second. Their results apply until the moment when the liquid phase reaches the second component of the boundary. By applying a similar procedure, Diaz, Fasano, Meirmanov [2] reduced the multidimensional one-phase Stefan problem to a spherically-symmetric one. After that, using Meirmanov's results [10], they obtained an estimate for the lifetime of the mushy region. They required the boundary temperature to be independent of spatial variables and increasing in time, but did not need smoothness of the boundary.

In the previous paper [3] we considered the Stefan problem in a domain with a $C^{1}$ smooth boundary. Using Green's function for the Dirichlet problem of the Laplace equation as a test function, we obtained several results concerning the behavior of the mush globally in time, for both the one- and two-phase Stefan problems. Here we generalize those results for the one-phase problem in a domain with a piecewisesmooth boundary. Moreover, we prove that the lifetime of the transient phase is uniformly bounded for every $t^{\prime}, \gamma>0$ on the Lebesgue set $\left\{G_{1}\left(t^{\prime}, x\right)<-\gamma\right\}$ of some specific function $G_{1}$. In the case of a $C^{2}$-smooth boundary and an appropriately smooth boundary temperature we obtain necessary and sufficient conditions for the disappearance of the mushy region in a finite time. In the third part of this paper we prove two results, which have been announced in [3] without a proof. They concern the behavior of a mush near the corner point of a two-dimensional domain. We conclude that the lifetime of a transient phase depends on the corner angle as follows: If it is greater than $\pi / 2$, then the lifetime is finite; otherwise, the mushy region exists for all times.

2. One-phase Stefan problem. We consider the Stefan problem in a bounded connected domain $\Omega \subset \mathbf{R}^{N}$ with a piecewise-smooth boundary $\Gamma$,

$$
\begin{gathered}
\frac{\partial U}{\partial t}=\Delta \boldsymbol{\Theta} \text { in } Q=\Omega \times(0, \infty), \\
\left.\Theta\right|_{\Gamma}=\boldsymbol{\Theta}^{1},\left.\quad U\right|_{t=0}=U_{0} .
\end{gathered}
$$

Henceforth $\Theta^{1} \in L^{\infty}(\Gamma \times(0, \infty)), U_{0} \in L^{\infty}(\Omega), \Theta=\chi(U) . \chi$ is an increasing Lipschitz-continuous function strongly monotonic everywhere except for the interval $U \in(-L, 0)$, where $\chi \equiv 0 . L$ is the latent heat. We shall use the inverse function $\Phi=\chi^{-1}$, which is given as a maximal monotone graph. The liquid phase corresponds to the set $U \geq 0$, the solid phase to the set $U \leq-L$, and the mushy zone to the set $U \in(-L, 0)$. By the mushy zone in physical variables we shall understand the set

$$
M=\{(x, t) \mid x \in \Omega, t \geq 0, U(x, t) \in(-L, 0)\}, \quad M\left(t_{0}\right)=M \cap\left\{t=t_{0}\right\} .
$$

We call a Stefan problem "one-phase", if either $U>-L$ or $U<0$ everywhere in $Q$. Without loss of generality, we consider only the first case. Let us define the 
auxiliary function $f_{\varepsilon}^{1}\left(x, x_{0}\right)$ for every $\varepsilon>0$ as a solution of the problem

$$
\begin{gathered}
\Delta_{x} f_{\varepsilon}^{1}\left(x, x_{0}\right)=\delta^{\varepsilon}\left(x-x_{0}\right), \\
\left.f_{\varepsilon}^{1}\left(x, x_{0}\right)\right|_{\Gamma}=0
\end{gathered}
$$

where

$$
\delta^{\varepsilon}=0 \quad \text { for }|y|>\varepsilon, \quad \delta^{\varepsilon}=1 /\left(\sigma_{N} \cdot \varepsilon^{N}\right) \quad \text { for }|y| \leq \varepsilon,
$$

and $\sigma_{N}$ is the measure of an $N$-dimensional unit ball.

The functions $\delta^{\varepsilon}$ converge to the delta-function $\delta$ weakly in the space of bounded measures as the parameter $\varepsilon$ tends to zero. Therefore, taking the limit $\varepsilon \rightarrow 0$, we observe that the function $f^{1}\left(x, x_{0}\right)=\lim _{\varepsilon \rightarrow 0} f_{\varepsilon}^{1}\left(x, x_{0}\right)$ is the Green's function for the Dirichlet problem. We assume that the function $f^{1}$ dependent on the geometry of the domain $\Omega$ and the point $x_{0}$ is known. Obviously, $f_{\varepsilon}^{1}\left(x, x_{0}\right)<0, f^{1}\left(x, x_{0}\right)<0$ for every $x, x_{0} \in \Omega$. The local estimates for elliptic problems (see [7]) yield the convergence of the normal derivatives with respect to the first argument $\left.\frac{\partial}{\partial n} f_{\varepsilon}^{1}\left(x, x_{0}\right)\right|_{\Gamma}$ to the function $\left.\frac{\partial}{\partial n} f^{1}\left(x, x_{0}\right)\right|_{\Gamma}$ in the norm of $L^{2}(\Gamma)$ for every $x_{0} \notin \Gamma$, as $\varepsilon \rightarrow 0$.

We shall use the following notation:

$$
\begin{aligned}
G_{1}\left(t, x_{0}\right) & =\int_{\Omega} U_{0}(x) f^{1}\left(x, x_{0}\right) d x-\int_{0}^{t} \int_{\Gamma} \frac{\partial}{\partial n} f^{1}\left(x, x_{0}\right) \Theta^{1}(x, t) d x d t, \\
\beta\left(t, x_{0}\right) & =\int_{\Omega} f^{1}\left(x, x_{0}\right) d x \cdot U_{\max }(t), \\
A\left(x_{0}\right) & =\left\{t \mid 0 \leq t<\infty, G_{1}\left(t, x_{0}\right)<\beta\left(t, x_{0}\right)\right\},
\end{aligned}
$$

where

$$
U_{\max }(t)=\max \left\{\sup _{x \in \Omega} U_{0}(x), \sup _{x \in \Gamma, 0 \leq \tau \leq t} \Phi\left(\boldsymbol{\Theta}^{1}(x, \tau)\right)\right\} .
$$

Due to the properties of Green's function, we conclude that the functions $G_{1}$ and $\beta$ are solutions of the following problems:

$$
\begin{gathered}
\Delta G_{1}(t, x)=U_{0}(x) \text { in } Q, \\
\left.G_{1}\right|_{\Gamma}=-\int_{0}^{t} \Theta^{1}(x, \tau) d \tau \\
\Delta \beta(t, x)=U_{\max }(t) \text { in } Q, \\
\left.\beta\right|_{\Gamma}=0 .
\end{gathered}
$$

Let us formulate now the first of the four main results of this study.

TheOREM 2.1. A solution of the one-phase Stefan problem (2.1), (2.2) with $U_{0}(x)>$ $-L, \Theta^{1}(x, t) \geq 0, U_{0}$ and $\Theta^{1}$ bounded possesses the following properties:

(i) For every $\gamma>0, t^{\prime}>0$ the mushy region disappears in a finite time $T_{*}$ on the set

$$
D=\left\{x \in M(0), G_{1}\left(t^{\prime}, x\right)<-\gamma\right\} .
$$

(ii) The lifetime $t_{*}\left(x_{0}\right)$ of the mush at the point $x_{0} \in M(0)$ may be estimated as follows :

$$
t_{*}\left(x_{0}\right) \leq \bar{t}\left(x_{0}\right)
$$


where $\bar{t}\left(x_{0}\right)=\inf \left\{t \mid t \in A\left(x_{0}\right)\right\}(\inf \varnothing=+\infty)$.

(iii) The inequality $\sup _{x \in M(0)} G_{1}(\infty, x)>0$ yields $|M(\infty)| \neq 0$.

Proof. Using the function $f_{\varepsilon}^{1}\left(x, x_{0}\right)$ as a test function for the problem (2.1), (2.2) in the domain $Q_{t}=\Omega \times(0, t)$ yields the equality

$$
\begin{aligned}
& \int_{\Omega} U(x, t) f_{\varepsilon}^{1}\left(x, x_{0}\right) d x-\int_{0}^{t} \int_{\Omega} \delta^{\varepsilon}\left(x-x_{0}\right) \Theta(x, \tau) d x d \tau \\
&=\int_{\Omega} U_{0}(x) f_{\varepsilon}^{1}\left(x, x_{0}\right) d x-\int_{0}^{t} \int_{\Gamma} \frac{\partial}{\partial n} f_{\varepsilon}^{1}\left(x, x_{0}\right) \Theta(x, \tau) d x d \tau .
\end{aligned}
$$

Using the definition of the functions $\delta^{\varepsilon}$ and the Lebesgue theorem, we conclude that the second integral in the left-hand side of this identity converges to the value $-\int_{0}^{t} \Theta\left(x_{0}, \tau\right) d \tau$ as $\varepsilon \rightarrow 0$ for a.e. $x_{0} \in \Omega$. Thus we have

$$
\int_{\Omega} U(x, t) f^{1}\left(x, x_{0}\right) d x-\int_{0}^{t} \Theta\left(x_{0}, \tau\right)=G_{1}\left(t, x_{0}\right) .
$$

Referring to the results of [4], we conclude that the mushy region $M(t)$ is a decreasing function in the set-theoretical sense, i.e., $\left|M\left(\tau_{1}\right) \backslash M\left(\tau_{2}\right)\right|=0$ for every $\tau_{1}>\tau_{2}$. Hence the equality $\Theta\left(x_{0}, \tau\right)=0$ is valid for a.e. $x_{0} \in M(t)$ if $\tau \leq t$. Therefore, we obtain

$$
\int_{\Omega} U(x, t) f^{1}\left(x, x_{0}\right) d x=G_{1}\left(t, x_{0}\right) \text { for a.e. } x_{0} \in M(t) .
$$

Let us prove next the first statement of Theorem 2.1. Using statement b) of Lemma A.1, we choose $\varepsilon>0$ such that

$$
\left|\int_{\Omega} f^{1}\left(x, x_{0}\right) d x\right| \leq \frac{\gamma}{\sup |U|}
$$

for every $x_{0} \in \omega_{\varepsilon}=\{x \in \Omega \mid \rho(x, \Gamma)<\varepsilon\}$. Then it follows from (2.6) that

$$
\gamma \geq\left|\int_{\Omega} U(x, t) f^{1}\left(x, x_{0}\right)\right|=\left|G_{1}\left(t, x_{0}\right)\right|>\gamma
$$

for every $t>t^{\prime}$ and a.e. $x_{0} \in M(t) \cap D \cap \omega_{\varepsilon}$.

The obtained contradiction implies nonexistence of the mushy region in the set $D \cap \omega_{\varepsilon}$ after time $t^{\prime}$ :

$$
M(t) \cap D \cap \omega_{\varepsilon}=\varnothing \quad \text { for } t \geq t^{\prime} .
$$

Finally, let us prove statement (i) for the set $D_{\varepsilon}=D \backslash \omega_{\varepsilon}$.

Consider first the case

(a) $\lim _{t \rightarrow \infty} G_{1}\left(t, x_{1}\right)=-\infty$ for some $x_{1} \in D_{\varepsilon}$.

Using Lemma A.1, we observe that in this case $G_{1}\left(t, x_{1}\right)$ tends to $-\infty$ uniformly in $D_{\varepsilon}$. Therefore, there exists such $t_{1}$ that the inequality

$$
\left|G_{1}(t, x)\right|>\sup _{x \in \Omega, t \geq 0}|U(x, t)| \cdot \sup _{x_{0} \in \Omega} \int_{\Omega}\left|f^{1}\left(x, x_{0}\right)\right| d x=C
$$

holds for $t \geq t_{1}, x \in D_{\varepsilon}$. Consequently, we have

$$
C \geq\left|\int_{\Omega} U(x, t) f^{1}\left(x, x_{0}\right) d x\right|=\left|G_{1}\left(t, x_{0}\right)\right|>C
$$


for $t>t_{1}$, a.e. $x_{0} \in M(t)$. The obtained contradiction proves the absence of the mushy region in the set $D$ after a finite time $t$ in this case.

(b) $G_{1}\left(\infty, x_{1}\right)>C_{1}$ for some $x_{1} \in D_{\varepsilon}$.

Using this condition, we note that the requirement of Lemma A.2 is fulfilled with $\Theta_{\infty}^{1} \equiv 0$. Let us take the sequence $\tau_{n}$ of Lemma A.2. Assume $\left|D_{\varepsilon} \cap M(t)\right| \neq 0$ for every $t \geq 0$. Then we may choose a sequence $x_{n} \in M\left(\tau_{n}\right) \cap D_{\varepsilon}$ such that $x_{n} \rightarrow x_{0}$ as $n \rightarrow \infty$. Applying the identity (2.6) for the points $\left(x_{n}, \tau_{n}\right)$ and taking the limit as $n \rightarrow \infty$, we find that

$$
0 \leq \int_{\Omega} U_{\infty}(x) f^{1}\left(x, x_{0}\right) d x=\lim _{n \rightarrow \infty} G_{1}\left(\tau_{n}, x_{n}\right) \leq-\gamma<0 .
$$

This contradiction implies that there exists a finite time $t_{2}$ after which the mushy region disappears from the set $D_{\varepsilon}$ in case (b).

Choosing $T_{*}=\max \left\{t^{\prime}, t_{1}, t_{2}\right\}$ completes the proof of statement (i).

The proof of the second statement of Theorem 2.1 directly follows from the estimates

$$
\begin{aligned}
& U(x, t) \leq \max \left\{\sup _{x \in \Omega} U_{0}(x), \sup _{0 \leq \tau \leq t, x \in \Gamma} \Phi\left(\Theta^{1}(x, \tau)\right)\right\} \\
& \beta\left(t, x_{0}\right) \leq \int_{\Omega} U(x, t) f^{1}\left(x, x_{0}\right) d x=G_{1}\left(t, x_{0}\right) \text { for } t<t_{*}\left(x_{0}\right),
\end{aligned}
$$

together with the definition of the set $A\left(x_{0}\right)$.

Let us turn now to the proof of statement (iii). The inequality in (iii) implies the existence of a point $x_{0} \in \Omega$ such that $G_{1}\left(\infty, x_{0}\right)>0$. Then the requirements of Lemma A.2 (with $\Theta_{\infty}^{1}=0,-L<U_{\infty}(x) \leq 0$ ) hold at the point $x_{0}$. Let us take the sequence $\tau_{n}$ of Lemma A.2. Applying the identity (2.5) to the points $\left(x_{0}, \tau_{n}\right)$ and taking a limit $n \rightarrow \infty$, we obtain

$$
\int_{\Omega} U(x, t) f^{1}\left(x, x_{0}\right) d x=\int_{0}^{\infty} \Theta\left(x_{0}, \tau\right) d \tau+G_{1}\left(\infty, x_{0}\right)>0 .
$$

Therefore, $U \not \equiv 0$, i.e., $|M(\infty)| \neq 0$. This completes the proof of Theorem 2.1.

In order to obtain a full criterion for disappearance of a mushy region in a finite time or its existence for all times, we need some additional requirements on the smoothness of $\Gamma$ and the function $\boldsymbol{\Theta}^{1}$ :

$$
\int_{0}^{t} \Theta^{1}(x, \tau) d \tau \in L^{\infty}\left(0, \infty ; C^{2}(\Gamma)\right), \quad \Gamma \in C^{2} .
$$

Let us define

$$
\begin{aligned}
f^{2}\left(x, x_{0}, \vec{\alpha}\right) & =\left(\nabla_{x_{0}} f^{1}\left(x, x_{0}\right), \vec{\alpha}\right), \\
G_{2}\left(t, x_{0}, \vec{\alpha}\right) & =\int_{\Omega} U_{0}(x) f^{2}\left(x, x_{0}, \vec{\alpha}\right) d x-\int_{0}^{t} \int_{\Gamma} \frac{\partial f^{2}}{\partial n}\left(x, x_{0}, \vec{\alpha}\right) \Theta^{1}(x, \tau) d x d \tau \\
& =\frac{\partial}{\partial \vec{\alpha}} G_{1}\left(t, x_{0}\right) .
\end{aligned}
$$

We will make use of the following function spaces: 
$C^{\beta}(\Omega)$-the space of Hölder-continuous functions with exponent $\beta \in[0,1)$,

$C^{1, \beta}(\Omega)$-the space of functions with first derivatives from $C^{\beta}(\Omega)$ $($ see [7]).

LEMMA 2.1. The following statements hold:

(a)

$$
G_{1} \in L^{\infty}\left(0, T ; C^{1, \beta}(\Omega)\right), \quad G_{2}(\cdot, \cdot, \vec{\alpha}) \in L^{\infty}\left(0, T ; C^{\beta}(\Omega)\right)
$$

for every $\beta \in[0,1), T>0$ and $\vec{\alpha} \in \mathbf{R}^{N}$.

(b) The conditions (2.7) yield

$$
G_{1} \in L^{\infty}\left(0, \infty ; C^{1, \beta}(\bar{\Omega})\right), \quad G_{2}(\cdot, \cdot, \vec{\alpha}) \in L^{\infty}\left(0, \infty ; C^{\beta}(\bar{\Omega})\right)
$$

for every $\beta \in[0,1)$ and $\vec{\alpha} \in \mathbf{R}^{N}$.

These conclusions follow from general estimates on the solutions of the Laplace equation with a bounded right-hand side (see [7]), the function $G_{1}$ being a solution of the problem (2.3).

THEOREM 2.2. Let the conditions (2.7) hold and $|M(0)| \neq 0$. Then the mushy region in the one-phase Stefan problem disappears in a finite time if and only if one of the following two conditions holds:

(i) $\max _{x \in \overline{M(0)}} G_{1}(\infty, x)<0$,

(ii) $\max _{x \in \overline{M(0)}} G_{1}(\infty, x)=0$, but $G_{1}(\infty, x)=0$ implies $x \in \Gamma$ and $G_{2}(\infty, x, \vec{\alpha})$ $>0$ for some outside direction $\vec{\alpha}$.

REMARK 1. Let us comment on condition (ii), namely

$G_{1}\left(\infty, x_{0}\right)=0$ and $G_{2}\left(\infty, x_{0}, \vec{\alpha}\right)>0$ for some $x_{0} \in \Gamma$ and some outside direction $\vec{\alpha}$.

As will be shown below, condition (ii) yields

$$
\int_{0}^{\infty} \Theta\left(x_{0}, \tau\right) d \tau=0, \quad-\int_{0}^{\infty} \frac{\partial}{\partial \alpha} \Theta\left(x_{0}, \tau\right) d \tau>0
$$

The last inequality implies that the overall heat flux into the thin boundary region near $x_{0}$ is positive and sufficient in order to melt all the material there in a finite time.

Proof. In what follows, it will be essential that the function $\theta(x, t)=\int_{0}^{t} \boldsymbol{\Theta}(x, \tau) d \tau$ is a solution of the problem

$$
\begin{gathered}
\Delta \theta(x, t)=U(x, t)-U_{0}(x) \text { in } Q, \\
\left.\theta\right|_{\Gamma}=\int_{0}^{t} \Theta^{1}(x, \tau) d \tau,
\end{gathered}
$$

and the right-hand side of (2.8a) is uniformly bounded in $t$. Consequently, $\theta(x, t) \in$ $L^{\infty}\left(0, \infty ; C^{1, \beta}(\bar{\Omega})\right)$ for every $\beta \in[0,1)$.

We prove next the sufficiency of each of the conditions (i), (ii) for disappearance of the mushy region in a finite time. Suppose that (i) is valid. Using Lemma 2.1, we obtain that $G_{1} \in L^{\infty}\left(0, \infty ; C^{1, \beta}(\bar{\Omega})\right)$ for every $\beta \in[0,1)$. Consequently, we may 
choose an instant $t^{\prime}>0$ such that $\max _{x \in \overline{M(0)}} G_{1}\left(t^{\prime}, x\right)<-\gamma$ for some $\gamma>0$. In this case the proof is merely a reformulation of assertion (i) of Theorem 2.1.

Let condition (ii) hold, but $|M(t)| \neq 0$ for every $t>0$. Using Lemma A.2, we can choose the sequence $\tau_{n} \rightarrow \infty$ such that $\Theta\left(x, \tau_{n}\right) \rightarrow 0, U\left(x, \tau_{n}\right) \rightarrow U_{\infty}(x)$ $\left(-L<U_{\infty} \leq 0\right)$ strongly in $L^{p}(\Omega)$ as $n \rightarrow \infty$ for every $p \in[1, \infty)$. At the same time, $G_{1}(\infty, x)<0$ for all $x \in \Omega \cap \overline{M(0)}$. With the help of Lemma 2.1 and statement (i) of Theorem 2.1, we obtain that $U_{\infty}(x)=0$ for every $x \in \Omega$. Therefore, we have the following convergences:

$$
\begin{aligned}
U\left(x, \tau_{n}\right) & \rightarrow 0 \text { as } n \rightarrow \infty \text { strongly in } L^{p}(\Omega), \quad p \in[1, \infty), \\
\theta\left(x, \tau_{n}\right) & \rightarrow-G_{1}(\infty, x) \text { as } n \rightarrow \infty \text { in } C^{\beta}(\bar{\Omega}), \\
\frac{\partial}{\partial \vec{\alpha}} \theta\left(x, \tau_{n}\right) & \rightarrow-G_{2}(\infty, x, \vec{\alpha}) \quad \text { as } n \rightarrow \infty \text { in } C^{\beta}(\bar{\Omega}),
\end{aligned}
$$

where $\beta \in[0,1)$.

Since it has been assumed that $|M(t)| \neq 0$ for each $t>0$, we can choose $x_{n}$ for every $n$ such that $\theta\left(x_{n}, t\right)=\frac{\partial}{\partial \bar{\alpha}} \theta\left(x_{n}, t\right)=0$ for every $t \leq \tau_{n}$. Choosing $x_{0}$ as a limit point of the sequence $x_{n}$ and using the convergence obtained above, we conclude that

$$
G_{1}\left(\infty, x_{0}\right)=G_{2}\left(\infty, x_{0}, \vec{\alpha}\right)=0 .
$$

However, these equalities contradict condition (ii).

Let us prove next the necessity of one of the conditions (i) or (ii) for disappearance of the mushy region in a finite time. Assume that there exists some instant $t_{*}$ such that $M(t)=\varnothing$ for $t \geq t_{*}$, i.e., $U(x, t) \geq 0$ for every $x \in \Omega, t \geq t_{*}$.

If $\max _{x \in \overline{M(0)}} G_{1}(\infty, x)>0$, then it follows from Theorem 2.1 that $|M(t)| \neq 0$ for every $t>0$. Therefore, $\max _{x \in \overline{M(0)}} G_{1}(\infty, x) \leq 0$. Whenever $\max _{x \in \overline{M(0)}} G_{1}(\infty, x)$ $<0$, condition (i) holds and hence the proof of Theorem 2.2 is completed. It remains to consider the case when $\max _{x \in \overline{M(0)}} G_{1}(\infty, x)=0$. This equality allows us to use Lemma A.2 $\left(\Theta_{\infty}^{1} \equiv 0\right)$ and, with the help of Lemma 2.1 , we may choose such a sequence $\tau_{n} \rightarrow \infty$ that ensures the convergences (2.9).

We note that solutions for which $\boldsymbol{\theta} \equiv 0$ should be excluded from consideration. Indeed, in this case $U(x, t) \equiv U_{0}(x)$ and hence $M(t) \equiv \varnothing$ for every $t>0$, which contradicts the condition $|M(0)| \neq 0$. Let us apply the strong maximum principle in the liquid phase $Q \backslash \bar{M}$, which is connected since $M$ is decreasing in time and disappears at $t_{*}$. Since $\Theta(x, t) \not \equiv 0$, it follows that for some instant $t_{0}>0$ the inequality $\Theta(x, t) \neq 0$ holds in the liquid phase in every section $t=$ const $\geq t_{0}$. Choosing $t_{1}=\max \left\{t_{0}, t_{*}\right\}$, we obtain $\Theta(x, t)>0$ for every $x \in \Omega, t \geq t_{1}$. Therefore $G_{1}(\infty, x)=-\theta(x, \infty)=-\int_{0}^{\infty} \Theta(x, \tau) d \tau<0$ for every $x \in \Omega$. Hence the function $G_{1}(\infty, x)$ may vanish only on the boundary $\Gamma$. Let $x_{0} \in \Gamma$ be such a point, so that $G_{1}\left(\infty, x_{0}\right)=0$. Then $G_{1}\left(\infty, x_{0}\right)=-\int_{0}^{\infty} \boldsymbol{\theta}\left(x_{0}, \tau\right) d \tau=0$. We consider the function $v(x)=\int_{t_{1}}^{\infty} \Theta(x, \tau) d \tau$, which is a solution of the problem

$$
\begin{aligned}
& \Delta v=-U\left(x, t_{1}\right), \\
& \left.v\right|_{\Gamma}=-\int_{t_{1}}^{\infty} \Theta(x, \tau) d \tau .
\end{aligned}
$$


Since $U\left(x, t_{1}\right)>0$ for every $x \in \Omega$ and $\left.v\right|_{\Gamma}>0$, we have $v(x)>0$ for every $x \in \Omega$; but $v\left(x_{0}\right)=0$, and, with the help of the strong maximum principle we conclude that $\partial v / \partial \vec{\alpha}\left(x_{0}\right)<0$ for every direction $\vec{\alpha}$ pointing outwards from $\Gamma$. Therefore we have

$$
\begin{aligned}
G_{2}\left(\infty, x_{0}, \vec{\alpha}\right) & =\frac{\partial}{\partial \vec{\alpha}} G_{1}\left(\infty, x_{0}\right)=-\frac{\partial}{\partial \vec{\alpha}} \theta\left(x_{0}, \infty\right) \\
& =-\frac{\partial}{\partial \vec{\alpha}} \int_{0}^{t_{1}} \boldsymbol{\Theta}\left(x_{0}, \tau\right) d \tau-\frac{\partial v}{\partial \vec{\alpha}}\left(x_{0}\right)>0,
\end{aligned}
$$

which completes the proof of Theorem 2.2.

REMARK 2. The results of [3] on the two-phase Stefan problem in a domain with a $C^{1}$-smooth boundary (Theorems 3.1,3.2, and Remark 1) hold also for problems in a domain with a piecewise-smooth boundary. The only change is that the decreased smoothness of the boundary requires the replacement of the condition (3.3) by (A.3) of Lemma A.2. This is necessary for convergence of the solution to the stationary one as $t \rightarrow \infty$ (see Lemma A.2).

3. Behavior of a mushy region near the corner point. Theorem 2.2 provides a sufficient and necessary condition for disappearance of the mush in a finite time on a domain with a smooth boundary. On the other hand, there is a gap in Theorem 2.1, applicable to a domain with a corner point, between the condition for disappearance of a mushy region (i) and the condition of its persistence for all times (iii). Indeed, the criteria (i), (iii) do not apply to the case $\sup _{x \in M(0)} G_{1}(\infty, x)=0$. If the function $G_{1}(\infty, x)$ achieves a zero supremum inside $\Omega$ or at a point where the boundary is smooth, it is possible to apply the technique of Theorem 2.2 and study the properties of the solution. In this case one only needs the local estimates in $C^{\beta}$ on the functions $G_{1}$ and $\Theta$, as the solutions of problems (2.3) and (2.8), respectively. Therefore, in principle the question is still open: what will happen if the function $G_{1}(\infty, x)$ achieves a zero supremum at some corner point $x_{0}$ ? Since $0=\left.G_{1}\left(t, x_{0}\right)\right|_{\Gamma}=-\int_{0}^{t} \boldsymbol{\Theta}^{1}\left(x_{0}, \tau\right) d \tau$, hence $\boldsymbol{\Theta}^{1}\left(x_{0}, \tau\right) \equiv 0$. For the sake of simplicity we assume that $\left.\Theta^{1}\right|_{\Gamma} \equiv 0$ in a neighborhood of the corner point, and the domain $\Omega$ is a sector of a two-dimensional circle.

Let $N=2, \vec{x}=\left(x_{1}, x_{2}\right)$. Let us consider the one-phase Stefan problem in the sector $\Omega_{\phi}=\left\{\left(r, \phi_{0}\right) \mid 0 \leq r \leq 1,0 \leq \phi \leq \phi_{0}\right\}$ with $r=\sqrt{x_{1}^{2}+x_{2}^{2}}, \phi=$ $\arctan \left(x_{1} / x_{2}\right)$. Let us set the boundary conditions

$$
\begin{gathered}
\left.\boldsymbol{\Theta}\right|_{\phi=0}=0,\left.\quad \boldsymbol{\Theta}\right|_{\phi=\phi_{0}}=0, \\
\left.\boldsymbol{\Theta}\right|_{r=1}=\boldsymbol{\Theta}^{1} \equiv \text { const }>0,
\end{gathered}
$$

and the initial data

$$
\left.U\right|_{t=0}=U_{0} \text {. }
$$

We formulate the following requirements on the initial data:

(i) $\Phi\left(\boldsymbol{\Theta}^{1}\right) \geq U_{0}>-L$, and there exists $r^{0} \in(0,1)$ such that $U_{0} \equiv l$ for $r<r^{0}, U_{0} \geq 0$ for $r \geq r^{0}, l \in(-L, 0)$; 
(ii) the function $U_{0}$ is nondecreasing with respect to the variables $x_{1}, x_{2}$ in the domain $\Omega_{\phi_{0} / 2} ;\left.\quad U_{0}\right|_{\phi=\phi_{1}}=\left.U_{0}\right|_{\phi=\phi_{0}-\phi_{1}}$ for $\phi_{1} \in\left[0, \phi_{0}\right]$.

THEOREM 3.1. Let the function $U_{0}$ satisfy the conditions (i), (ii), $U$ be a solution of the Stefan problem with the initial-boundary data (3.1), (3.2) and $0<\phi_{0} \leq \pi / 2$. Then there exists a decreasing positive function $r_{0}$ such that $U \equiv l$ for $0 \leq r \leq$ $r_{0}(t), t \geq 0$, i.e., $M(t) \supset \Omega_{\phi_{0}} \cap\left\{r<r_{0}(t)\right\}, t \geq 0$.

Proof. Symmetry of the initial data about the line $\phi=\phi_{0} / 2$ (see (ii)) allows us to consider the problem in the domain $\Omega_{\phi_{0} / 2}$ with the additional boundary data

$$
\left.\frac{\partial \boldsymbol{\Theta}}{\partial \phi}\right|_{\phi=\phi_{0} / 2}=0
$$

With the help of the standard methods for the Stefan problem one can prove that the function $U$ satisfies the conditions (ii) for all times.

It follows from the results of [4] that the mushy region $M(t)$ is decreasing in time; inside this region $U \equiv l$, whereas on the outside $U \geq 0$. Assume that the statement of Theorem 3.1 does not hold for some $t=t_{0}$. Then there exists a sequence of

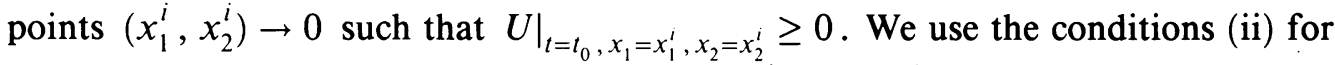
$\left.U\right|_{t=t_{0}}$ and conclude that $\left.U\right|_{t=t_{0}} \geq 0$ for $x_{1} \geq x_{1}^{i}, x_{2} \geq x_{2}^{i}$ in $\Omega_{\phi_{0} / 2}$, i.e., $\left.U\right|_{t=t_{0}} \geq 0$ in the entire domain $\Omega$. Therefore $t_{0} \neq 0$.

Choose the minimum value $t_{0}$ with the above property. There exists a decreasing function $r_{0}(t)$ on the interval $\left[0, t_{0}\right)$ described in Theorem 3.1. Let us define the function $f=\left(1 / r^{\gamma}-r^{\gamma}\right)$ and the domain $\Omega_{\phi_{0} / 2}^{\varepsilon}=\Omega_{\phi_{0} / 2} \cap\left\{r>r_{0}\left(t_{0}-\varepsilon\right)\right\}$, where $\gamma=$ $\pi / \phi_{0}, \varepsilon>0$. Testing Eq. (2.1) with the function $f$ over the domain $\Omega_{\phi_{0} / 2}^{\varepsilon} \times\left(0, t_{0}-\varepsilon\right)$ we obtain

$$
\left.\int_{\Omega_{\phi_{0} / 2}^{\varepsilon}} f \cdot U d \vec{x}\right|_{t=0} ^{t=t_{0}-\varepsilon}=-\int_{0}^{t_{0}-\varepsilon} \int_{r=1} \frac{\partial f}{\partial n} \Theta^{1} d x d \tau
$$

Continuity of the function $U$ in the norm $L^{1}(\Omega)$ over the section $t=$ const yields

$$
\int_{\Omega_{\phi_{0} / 2}}\left|U\left(x, t_{0}-\varepsilon\right)-U\left(x, t_{0}\right)\right| d x \rightarrow 0 \text { as } \varepsilon \rightarrow 0
$$

(in particular, this result easily follows from those of [4]). From here, we deduce that the integral in the left-hand side of (3.3) converges to the value

$$
I=\lim _{\delta \rightarrow 0} I^{\delta}=\left.\int_{\{r>\delta\} \cap \Omega_{\phi_{0} / 2}} f \cdot U d x\right|_{t=0} ^{t=t_{0}} \quad \text { as } \varepsilon \rightarrow 0 .
$$

The functions $f$ and $U$ are bounded for $r \geq r_{0}(0)$. Using the inequality $\gamma=$ 
$\pi / \phi_{0} \geq 2$, we can estimate the integral $I^{\delta}$ as follows:

$$
\begin{aligned}
\left|I^{\delta}\right| & =\left|\int_{\left\{\delta<r<r^{0}\right\} \cap \Omega_{\phi_{0} / 2}} f \cdot U d x\right|_{t=0}^{t=t_{0}}+\left.\int_{\left\{r^{0}<r<1\right\} \cap \Omega_{\phi_{0} / 2}} f \cdot U d x\right|_{t=0} ^{t=t_{0}} \mid \\
& \geq\left|\int_{\left\{\delta<r<r^{0}\right\} \cap \Omega_{\phi_{0} / 2}} f \cdot U d x\right|_{t=0}^{t=t_{0}}|-C=| \int_{\left\{\delta<r<r^{0}\right\} \cap \Omega_{\phi_{0} / 2}} f \cdot\left(U\left(x, t_{0}\right)-l\right) d x \mid-C \\
& \geq|l| \int_{\delta}^{r^{0}}\left(r^{1-\gamma}-r^{1+\gamma} d r\right) \cdot \int_{0}^{\phi_{0} / 2} \sin (\gamma \phi) d \phi-C \geq C_{1} g^{\gamma}(\delta)-C,
\end{aligned}
$$

where

$$
g^{\gamma}(\delta)=\left\{\begin{array}{ll}
\delta^{2-\gamma} & \text { for } \gamma>2 \\
|\ln \delta| & \text { for } \gamma=2 .
\end{array} .\right.
$$

Hence $\lim _{\delta \rightarrow 0}\left|I^{\delta}\right|=|I|=\infty$ for $\gamma \geq 2$. This conclusion contradicts the previous assumption, since the right-hand side of (3.3) is bounded. Therefore, $r_{0}(t)>0$ for every $t>0$, which completes the proof of Theorem 3.1.

REMARK 3. Using the solution constructed above as an upper majorant, one may prove correctness of the results of Theorem 3.1 for a solution of the Stefan problem with initial-boundary data (3.1), (3.2), where $\Theta^{1}$ is a bounded measurable positive function and $U_{0}$ satisfies condition (i) only.

In addition to Theorem 3.1 we formulate the following result:

Theorem 3.2. Let $U_{0}$ satisfy (i) and $\phi_{0} \in(\pi / 2,2 \pi]$. Then the lifetime $t_{*}$ of a mushy region in the Stefan problem (2.1), (3.1), (3.2) is estimated as

$$
t_{*} \leq \frac{\max \left\{L, \Phi\left(\Theta^{1}\right)\right\}}{2(2-\gamma) \Theta^{1}} \text { where } \gamma=\pi / \phi_{0} .
$$

Proof. Let us change the variables from $\left(x_{1}, x_{2}\right)$ to $(v, w)$ defined as

$$
v=r^{\gamma} \sin \gamma \phi, \quad w=r^{\gamma} \cos \gamma \phi .
$$

The angle $0 \leq \phi \leq \phi_{0}$ transforms into the semiplane $v \geq 0$, the sector of a circle $\left\{r=1,0 \leq \phi \leq \phi_{0}\right\}$ into the semicircle $\left\{v^{2}+w^{2}=1, v>0\right\}$. The equation of heat diffusion can be rewritten as

$$
U_{t}-\gamma^{2}\left(v^{2}+w^{2}\right)^{(\gamma-1) / \gamma}\left(\boldsymbol{\Theta}_{v v}+\boldsymbol{\Theta}_{w w}\right)=0,
$$

the initial-boundary data transform into

$$
\begin{aligned}
\left.U\right|_{t=0} & =U_{0}(v, w), \\
\left.\Theta\right|_{v \geq 0, v^{2}+w^{2}=1} & =\boldsymbol{\Theta}^{1},\left.\quad \boldsymbol{\Theta}\right|_{v=0}=0 .
\end{aligned}
$$

For the problem (3.5), (3.6) we construct the one-dimensional lower majorant $\underline{U}(v, t)$ as a solution of the following problem:

$$
\begin{gathered}
\underline{U}_{t}-\gamma^{2} v^{2(\gamma-1) / \gamma} \underline{\boldsymbol{\Theta}}_{v v}=0, \\
\underline{U}(0, v)=\underline{U}_{0}(v) \quad \text { for } 0<v<1, \\
\underline{\boldsymbol{\Theta}}(0, t)=0, \quad \underline{\boldsymbol{\Theta}}(1, t)=\boldsymbol{\Theta}^{1} \quad \text { for } t \geq 0,
\end{gathered}
$$


where

$$
\underline{U}_{0}(v)= \begin{cases}-L & \text { for } 0<v<\left(r^{0}\right)^{\gamma} ; \\ 0 & \text { for }\left(r^{0}\right)^{\gamma}<v<1 .\end{cases}
$$

This is a one-phase Stefan problem with a nonconstant heat conductivity $\gamma^{2} v^{2(\gamma-1) / \gamma}$.

Using the standard methods (see for example [11]), we may prove the existence of a classical solution to this problem on some interval $\left(0, t_{0}\right)$, i.e., there exists a smooth decreasing function $v=R(t)$ such that $\underline{U} \equiv-L$ for $v<R(t), \underline{U} \geq 0$ for $v \geq R(t), R(0)=r_{0}^{\gamma} ; R\left(t_{0}\right)=0$, or $t_{0}=\infty$. Besides, it may easily be shown that $\underline{\boldsymbol{\theta}}_{v v}$ is a nonnegative distribution, and consequently

$$
\begin{aligned}
& (U-\underline{U})_{t}-\gamma^{2}\left(v^{2}+w^{2}\right)^{(\gamma-1) / \gamma} \Delta_{(v, w)}(\boldsymbol{\Theta}-\underline{\boldsymbol{\theta}}) \\
& =\gamma^{2}\left(\left(v^{2}+w^{2}\right)^{(\gamma-1) / \gamma}-v^{2(\gamma-1) / \gamma)}\right) \underline{\boldsymbol{\Theta}}_{v v} \geq 0 .
\end{aligned}
$$

Here

$$
\Delta_{(v, w)}=\frac{\partial^{2}}{\partial v^{2}}+\frac{\partial^{2}}{\partial w^{2}} .
$$

Since the initial-boundary data for $\underline{U}$ have been chosen such that

$$
\begin{array}{ll}
U-\underline{U} \geq 0 & \text { for } t=0, v^{2}+w^{2}<1, v>0, \\
\Theta-\underline{\Theta} \geq 0 & \text { for } t>0, v^{2}+w^{2}=1, v>0,
\end{array}
$$

we obtain

$$
U \geq \underline{U} \text { for } t \geq 0, v^{2}+w^{2} \leq 1, v \geq 0 .
$$

Let us define the lifetime of the mush for the problem (3.7), (3.8) as

$$
t_{0}=\inf \{t \mid \underline{U}(t, v) \geq 0 \text { for a.e. } v \in(0,1)\} .
$$

Then $\left.U\right|_{t=t_{0}} \geq\left.\underline{U}\right|_{t=t_{0}} \geq 0$ and the mushy region for the solution of problem (2.1) (3.1) does not exist for time $t \geq t_{0}$. Hence we have an estimate for the lifetime of the mushy region for the problem (2.1) (3.1), namely

$$
t_{*} \leq t_{0} \text {. }
$$

In order to estimate $t_{0}$ we multiply Eq. (3.7) by $(1-v) v^{(2-2 \gamma) / \gamma}$ and integrate it over the domain $(0,1) \times(0, t), t<t_{0}$ :

$$
\int_{0}^{1}(1-v) v^{(2-2 \gamma) / \gamma}(\underline{U}(v, t)-\underline{U}(v, 0)) d v=\gamma^{2} \boldsymbol{\Theta}^{1} t .
$$

Estimating the left-hand side of the equality, we obtain

$$
\frac{\max \left(L, \Phi\left(\Theta^{1}\right)\right)}{2(2-\gamma) \Theta^{1}} \geq t \text { for every } t<t_{0},
$$

which implies that this inequality holds also for $t=t_{0}$. Since $t_{*} \leq t_{0}$, this completes the proof of Theorem 3.2.

Acknowledgment. We are grateful to J. R. Ockendon, I. Rubinstein, and J. C. W. Rogers for valuable remarks that helped us to make the paper clearer. 
Appendix. Henceforth we assume $\Theta^{1} \in L^{\infty}(\Gamma \times(0, \infty)), U_{0} \in L^{\infty}(\Omega)$, and $\Gamma$ to be a piecewise-smooth boundary.

Lemma A.1. (a) The function $I(x, t)=\int_{0}^{t} \int_{\Gamma} f^{1}(y, x)\left|\Theta^{1}(y, \tau)\right| d y d \tau$ satisfies the inequality

$$
A^{-1} I\left(x_{2}, t\right) \leq I\left(x_{1}, t\right) \leq A I\left(x_{2}, t\right)
$$

for every $t>0, x_{1}, x_{2} \in \Omega$, where the positive constant $A$ depends only on the distance from the points $x_{1}, x_{2}$ to the boundary $\Gamma$.

(b) The function $u(x)=\int_{\Omega} f^{1}(y, x) d y$ belongs to $C^{\beta}(\bar{\Omega})$ for every $\beta \in[0,1)$ and $u(x)=0$ for $x \in \Gamma$.

Proof. Let us take arbitrary points $x_{1}, x_{2} \in \Omega$. Choose $\varepsilon>0$ such that $\varepsilon$ neighborhoods $B_{\varepsilon}\left(x_{1}\right), B_{\varepsilon}\left(x_{2}\right)$ of points $x_{1}, x_{2}$ are included in the domain $\Omega$. The functions $f^{1}\left(x, x_{1}\right), f^{1}\left(x, x_{2}\right)$ satisfy the Laplace equation in the domain $\Omega_{1}=$ $\Omega \backslash\left(B_{\varepsilon}\left(x_{1}\right) \cup B_{\varepsilon}\left(x_{2}\right)\right)$ so that

$$
A_{\text {min }} g(x) \leq f^{1}\left(x, x_{1}\right) \leq A_{\max } g(x), \quad i=1,2
$$

for every $x \in \Omega$, where

$$
\begin{aligned}
& A_{\max }=\max _{x \in \partial\left(B_{\varepsilon}\left(x_{1}\right) \cup B_{\varepsilon}\left(x_{2}\right)\right)}\left\{f^{1}\left(x, x_{1}\right), f^{1}\left(x, x_{2}\right)\right\}, \\
& A_{\min }=\min _{x \in \partial\left(B_{\varepsilon}\left(x_{1}\right) \cup B_{\varepsilon}\left(x_{2}\right)\right)}\left\{f^{1}\left(x, x_{1}\right), f^{1}\left(x, x_{2}\right)\right\} .
\end{aligned}
$$

The function $g$ is a solution of the problem

$$
\begin{gathered}
\Delta g=0 \quad \text { in } \Omega_{1}, \\
\left.g\right|_{\Gamma}=0,\left.\quad g\right|_{\partial\left(B_{\varepsilon}\left(x_{1}\right) \cup B_{\varepsilon}\left(x_{2}\right)\right)}=1 .
\end{gathered}
$$

With the help of (A.2), we obtain the inequality

$$
\begin{aligned}
I\left(x_{1}, t\right) & \leq A_{\min } \int_{0}^{t} \int_{\Gamma} \frac{\partial g}{\partial n}(y)\left|\Theta^{1}(y, \tau)\right| d y d \tau \\
& \leq \frac{A_{\min }}{A_{\max }} \int_{0}^{t} \int_{\Gamma} \frac{\partial f^{1}}{\partial n}\left(y, x_{2}\right)\left|\boldsymbol{\Theta}^{1}(y, \tau)\right| d y d \tau=A I\left(x_{2}, t\right) .
\end{aligned}
$$

The exchange of variables $x_{1}$ and $x_{2}$ in this inequality completes the proof of the statement (a).

The statement (b) follows immediately from the properties of the function $u(x)$ (see [7]), which is a solution of the problem

$$
\Delta u=1 \quad \text { in } \Omega,\left.\quad u\right|_{\Gamma}=0 .
$$

Lemma A.2. Let $\Theta^{1} \in L^{\infty}(\Gamma \times(0, \infty)), U_{0} \in L^{\infty}(\Omega)$,

$$
\int_{0}^{\infty}\left|\Theta^{1}(x, t)-\Theta_{\infty}^{1}(x)\right| \frac{\partial f^{1}}{\partial n}\left(x, x_{0}\right) d x d t<C
$$


for some $x_{0} \in \Omega$. Then there exists a sequence $\tau_{n} \rightarrow \infty$ such that the following convergences hold for the solution of the problem (2.1), (2.2):

$$
\begin{aligned}
& \Theta\left(x, \tau_{n}\right) \rightarrow \Theta_{\infty}(x) \text { strongly in } L^{p}(\Omega), \\
& U\left(x, \tau_{n}\right) \rightarrow U_{\infty}(x) \text { weakly* in } L^{\infty}(\Omega)
\end{aligned}
$$

as $n \rightarrow \infty$ for every $p \in[1, \infty)$. Here the function $\Theta_{\infty}$ is a solution of the problem

$$
\begin{aligned}
& \Delta \Theta_{\infty}=0 \text { in } \Omega, \\
& \left.\Theta_{\infty}\right|_{\Gamma}=\Theta_{\infty}^{1},
\end{aligned}
$$

and the function $U_{\infty}$ satisfies the equation of state $\Theta_{\infty}(x)=\chi\left(U_{\infty}(x)\right)$.

For the one-phase Stefan problem $\left(U_{0}>-L, \Theta^{1} \geq 0\right), U\left(x, \tau_{n}\right) \rightarrow U_{\infty}(x)$ strongly in $L^{p}(W)$ for any $p \in[1, \infty)$.

Proof. Let us recall a procedure, used first by Kamenomostskaya [6]. Taking the difference of integral equalities for the problems (2.1), (2.2), and (A.4), we obtain

$$
\begin{aligned}
& \int_{0}^{t} \int_{\Omega}\left[\phi_{t}\left(U(x, \tau)-U_{\infty}(x)\right)+\Delta \phi\left(\Theta(x, \tau)-\Theta_{\infty}(x)\right)\right] d x d \tau \\
& \quad=\frac{\partial \phi}{\partial n}\left(\Theta^{1}(x, \tau)-\Theta_{\infty}^{1}(x)\right) d x d \tau-\int_{\Omega} \phi(x, 0)\left(U_{0}(x)-U_{\infty}(x)\right) d x,
\end{aligned}
$$

where $\phi \in W_{2}^{2,1}\left(Q_{t}\right)$ and $\phi=0$ for $\tau=t$ or $x \in \Gamma$.

We consider the following auxiliary problem:

$$
\begin{gathered}
\phi_{t}^{\varepsilon}+(\mu(x, \tau)+\varepsilon) \Delta \phi^{\varepsilon}=(\mu(x, t)+\varepsilon) S^{\gamma}(x, \tau) \text { in } Q_{t}, \\
\left.\phi^{\varepsilon}\right|_{\tau=t}=0,\left.\quad \phi^{\varepsilon}\right|_{\Gamma}=0,
\end{gathered}
$$

where

$$
\mu(x, \tau)=\left(\boldsymbol{\Theta}(x, \tau)-\boldsymbol{\Theta}_{\infty}(x)\right) /\left(U(x, \tau)-U_{\infty}(x)\right)
$$

is a bounded nonnegative function and

$$
S^{\gamma}(x, \tau)= \begin{cases}\operatorname{sign}\left(\Theta(x, \tau)-\Theta_{\infty}(x)\right) & \text { for } x \in \Omega^{\gamma}=\{y \mid \rho(y, \Gamma) \geq \gamma\}, \\ 0 & \text { for } x \in \Omega \backslash \Omega^{\gamma},\end{cases}
$$

for positive $\gamma \leq \frac{1}{2} \rho\left(x_{0}, \Gamma\right)$. The following statements hold:

$$
\begin{gathered}
\sqrt{\mu+\varepsilon} \Delta \phi^{\varepsilon} \in L^{2}\left(Q_{t}\right), \quad \phi^{\varepsilon} \in L^{2}\left(Q_{t}\right), \\
\nabla \phi^{\varepsilon} \in L^{\infty}\left(0, t ; L^{2}(\Omega)\right) \quad \text { uniformly over } \varepsilon>0, \\
u^{-}(x) \leq \phi^{\varepsilon}(x, t) \leq u^{+}(x) .
\end{gathered}
$$

Here the majorants $u^{-}$and $u^{+}$are solutions of the problems

$$
\begin{gathered}
-\Delta u^{ \pm}= \begin{cases} \pm 1 & \text { in } \Omega^{\gamma}, \\
0 & \text { in } \Omega \backslash \Omega^{\gamma},\end{cases} \\
\left.u^{ \pm}\right|_{\Gamma}=0 .
\end{gathered}
$$


Moreover, by analogy with Lemma A.1, we can estimate $\frac{\partial \phi^{\varepsilon}}{\partial n}$ as

$$
\left|\frac{\partial \phi^{\varepsilon}}{\partial n}\right| \leq C(\gamma) \frac{\partial f^{l}}{\partial n} .
$$

Using $\phi^{\varepsilon}$ as a test function we obtain

$$
\begin{gathered}
\int_{0}^{t} \int_{\Omega}\left(U(x, \tau)-U_{\infty}(x)\right)(\mu(x, \tau)+\varepsilon) S^{\gamma}(x, \tau) d x d \tau \\
\quad-\int_{0}^{t} \int_{\Omega} \varepsilon \Delta \phi^{\varepsilon}\left(U(x, \tau)-U_{\infty}(x)\right) d x d \tau \\
\leq \int_{0}^{t} \int_{\Gamma} \frac{\partial f^{1}}{\partial n}\left(x, x_{0}\right)\left|\Theta^{1}(x, \tau)-\Theta_{\infty}^{1}(x)\right| d x d \tau \\
\quad+\int_{\Omega} \max \left\{\left|u^{+}(x)\right|,\left|u^{-}(x)\right|\right\}\left|U_{0}(x)-U_{\infty}(x)\right| d x \\
\leq C_{1}(\gamma) .
\end{gathered}
$$

It follows from statements (A.5) that the second integral in the left-hand side of the inequality converges to zero as $\varepsilon \rightarrow 0$; so we have

$$
\int_{0}^{t} \int_{\Omega^{i}}\left|\boldsymbol{\Theta}(x, \tau)-\boldsymbol{\Theta}_{\infty}(x)\right| d x d \tau<C_{1}(\gamma),
$$

where $C_{1}(\gamma)$ may be chosen independent of $t$. With the help of this inequality, which is satisfied for any $\gamma>0$, we may find the sequence $\tau_{n} \rightarrow \infty$ such that $\Theta\left(x, \tau_{n}\right) \rightarrow \Theta_{\infty}(x)$ strongly in $L^{p}(\Omega)$ for any $p \in[1, \infty)$. At the same time, the existence of the function $U_{\infty}(x)$, such that $U\left(x, \tau_{n}\right) \rightarrow U_{\infty}(x)$ weakly* in $L^{\infty}(\Omega)$ and $\Theta_{\infty}(x)=\chi\left(U_{\infty}(x)\right)$, follows from the monotonicity of the function $\chi$.

In the one-phase case $(U(x, t)>-L)$ we divide the function $U$ into a positive and negative part: $U^{+}=\max \{U, 0\}, U^{-}=\min \{U, 0\}$. The function $U^{+}=$ $(\Phi(\Theta))^{+}$is a continuous function of the temperature $\boldsymbol{\Theta}(x, t)$. Thus $U^{+}\left(x, \tau_{n}\right) \rightarrow$ $U_{\infty}^{+}(x)$ as $n \rightarrow \infty$ strongly in $L^{p}(\Omega)$. It follows from the results of [4] that $U^{-}(x, \tau) \equiv U_{0}(x)$ in $M$ and $U^{-}(x, t) \equiv 0$ in the liquid phase. Since the set $M(t)$ does not increase in time, the function $U^{-}(x, t)$ increases in time. Therefore, $U^{-}\left(x, \tau_{n}\right) \rightarrow U_{\infty}^{-}(x)$ strongly in $L^{p}(\Omega)$. This completes the proof of Lemma A.2.

\section{REFERENCES}

[1] D. R. Atthey, A finite difference scheme for melting problems, J. Inst. Math. Appl. 354 (1975)

[2] J. I. Diaz, A. Fasano, and A. Meirmanov, On the disappearance of the mushy region in multidimensional Stefan problem, Proc. Free Boundary Problems: Theory \& Applications, Montreal, 1990

[3] I. G. Götz and B. Zaltzman, On the behavior of mushy region in a Stefan problem, Internat. Ser. Numer. Math. 9, 155-163 (1991)

[4] I. G. Götz and B. Zaltzman, Nonincrease of mushy region in a nonhomogeneous Stefan problem, Quart. Appl. Math. XLIX, 741-746 (1991)

[5] B. Gustafsson and J. Mossino, Quelques inequalités isopérimétriques pour le problème de Stefan, Math. Analysis, C. R. Acad. Sci. Paris 305 (1), 669-672 (1987)

[6] S. L. Kamenomostskaya, On the Stefan problem, Mat. Sbornik. 53 (95), 489-514 (1961) (in Russian)

[7] O. A. Ladyženskaja and N. N. Ural'ceva, Linear and quasilinear equations of elliptic type, Nauka: Moscow (1967) (in Russian) 
[8] A. M. Meirmanov, An example of nonexistence of a classical solution of the Stefan problem, Soviet Math. Dokl. 3 (28), 564-566 (1981)

[9] A. M. Meirmanov, The structure of a generalized solution of the Stefan problem, Periodic solutions, Dokl. Akad. Nauk SSSR 272 (4), 789-791 (1981); English transl. in Soviet Math. Dokl. 2 (28) 440-443 (1983)

[10] A. M. Meirmanov, On the disappearance of the mushy region in the Stefan problem with spherical symmetry (in Russian), Dinamika sploshnoi sredy, Lavrent' ev Institute of Hydrodynamics 91, 86-99 (1985)

[11] A. M. Meirmanov, The Stefan problem, De Gruyter Expositions in Math., Berlin, 244 (1992)

[12] O. A. Oleinik, A method of solution of the general Stefan problem, Soviet Math. Dokl. 1, 1350-1354 (1960)

[13] M. Primicerio, Mushy region in phase-change problem, Appl. Nonlinear Funct. Anal., Lang, Frankfurt/Main, 251-269 (1982)

[14] J. C. W. Rogers and A. E. Berger, Some properties of the nonlinear semigroup for the problem $u_{t}-D f(u)=0$, Nonlinear Anal., Theory, Methods, and Applications 8 (8), 909-939 (1984) 